\title{
Development of Long-Acting Injectable Ketamine Loaded PLGA Microparticles as a Non-opioid Analgesic
}

Juma N. Daniels ${ }^{1}$ and Andrew Otte ${ }^{2}$

${ }^{1}$ Indiana University School of Medicine; ${ }^{2}$ Purdue University School of Biomedical Engineering

\section{Background/Objective:}

Ketamine, a psychedelic, is a noncompetitive N-methyl-D-aspartate receptor antagonist that may also bind to mu opioid receptors. Historically, it has been used as an anesthetic (Ketalar ${ }^{\circledR}$ ), although now has found uses as a novel, quick acting, antidepressant for treatment-resistant depression (Spravator ${ }^{\circledR}$ ) and could be used as an adjuvant to opioid analgesia providing opioidsparing effects. One major advantage over opioids is Ketamine does not suffer from respiratory depression and maintains patent airways during anesthesia. Ketamine is only available as a short-acting injectable solution or a nasal spray. Our goal is to develop a long-acting injectable form in a biodegradable matrix poly(lactic-co-glycolic) acid (PLGA) that does not have a burst release and provides 5-7 days of steady-state plasma levels.

\section{Methods:}

A mechanistic approach towards development of a long-acting injectable began with a solubility screen of Ketamine. Based on these results, experiments began with an oil in water emulsification with two theoretical drug loadings (25\% and $40 \%)$ and two processing conditions - (1) aqueous extraction and (2) aqueous extraction, intermediate drying, and a 25\% Ethanol wash. The formulations were characterized for drug loading, drug release, and crystallinity and imaged using scanning electron microscopy (SEM).

\section{Results:}

Minimal differences were noted in the release profiles between formulations. Although, a significant difference was noted between the two processing conditions, where the extra intermediate drying step and $25 \%$ ethanol wash resulted in a significant slowing of the drug release rate.

\section{Conclusion and Implications:}

The difference in release kinetics is hypothesized to be due to densification of the PLGA matrix, based on the increase in surface roughness/wrinkling in the SEM images, crystallinity increase, and on their respective powder x-ray diffraction patterns. Our preliminary results demonstrate the feasibility of a longer acting Ketamine using PLGA. Further refinement of these formulations and rodent pharmacokinetic studies will be done in future. 